\title{
Monitoring of Radiation Fields in Near Earth Space and Atmosphere in New Space Projects of Moscow University
}

\author{
Sergey Svertilov ${ }^{1,2 *}$, Viktor Bengin ${ }^{1}$, Vitaly Bogomolov ${ }^{1,2}$, Gali Garipov ${ }^{1}$, Mikhail \\ Dobynde $^{1}$, Ivan Zolotarev ${ }^{1}$, Vladimir Kalegaev ${ }^{1}$, Pavel Klimov ${ }^{1}$, Vladislav Osedlo ${ }^{1}$, Mikhail \\ Panasyuk $^{1,2}$, Oleg Peretjat'ko', Vasily Petrov' ${ }^{1}$, Mikhail Podzolko ${ }^{1}$ \\ ${ }^{1}$ Lomonosov Moscow State University, D.V. Skobeltsyn Institute of Nuclear Physics, 1(2), \\ Leninskie gory, GSP-1, Moscow 119991, Russian Federation \\ ${ }^{2}$ Lomonosov Moscow State University, Physics Department, Leninskie Gory, Moscow 119991, \\ Russia
}

\begin{abstract}
The Universat-SOCRAT project is developed in the Moscow State University aiming to forecast space-related risks for aviation, suborbital, and orbital flights and provide new knowledge on the magnetosphere and atmosphere of the Earth. An essential part of the system is a multi-satellite constellation, which would operate in the lowEarth orbit. Among other things, it would monitor the radiation and magnetic-wave environment in the vicinity of the Earth: in space and atmosphere. An Earth observation system, which operates in gamma and visible spectral range, should allow attribute detected changes in the environment to the atmospheric phenomena. We have already designed the instruments to detect increases in the flux of energetic charged particles (solar energetic particles, galactic cosmic rays, and electrons precipitating from radiation belts), geomagnetic disturbances, and electromagnetic transients in the atmosphere. The first stage of the program started on July 5, 2019, with a successful launch of three $3 \mathrm{U}$ CubeSats from the Vostochny cosmodrome. These satellites carry instruments for monitoring space radiation and prototype of the device for observing the Earth's atmosphere in the ultraviolet range. The collected data has confirmed the advantages of multi-satellite observations for the goals of the project. During this year, we plan to launch two more 6U CubeSats with charged particle and gamma-ray detectors, magnetometers, and instrument for detecting of atmospheric electromagnetic transients. We suppose that these satellites will lay the foundation of the space threat monitoring system.
\end{abstract}

\section{Introduction}

As it is well-known, space weather monitoring implies two aspects. The first is fundamental, which addresses the solar-terrestrial and atmospheric-ionospheremagnetospheric relations and interactions. The second is the application aspect, which is

* Corresponding author: $\underline{\text { sis@,coronas.ru }}$ 
related to the study and predicting the effect of space radiation on the spacecraft and aviation systems and astronauts health. Physical processes in the magnetosphere of the Earth determine radiation particle fluxes. Radiation conditions in near-Earth space are determined by physical processes in the space environment. In this case, the input parameters for predicting the level of the radiation background on the near-Earth orbits are the values of high-energy charged particle fluxes in near-Earth space. Although under quiet geo-heliomagnetic conditions radiation background is mainly due to galactic cosmic rays (GCR), charged particle fluxes are characterized by significant medium and long-term variations, which cannot be described by the existing quasi-static models of the Earth's radiation belts. Solar influence and precipitation of particles from the Earth's radiation belts in the atmosphere are the main factors that should be taken into account to predict the radiation environment in the near-Earth space. During the so-called "extreme events", the radiation background caused by cosmic rays can increase significantly due to solar energetic particles (SEP). SEPs are mainly protons and ions accelerated to high energies on shock waves generated by coronal mass ejections in the solar corona and interplanetary environment during powerful solar flares. SEP events can last from tens of minutes to several days. Strong SEP events comprise a significant number of particles with energies of more than $400 \mathrm{MeV}$, they can generate secondary neutrons in the atmosphere, increasing the background radiation level even at aircraft altitudes. A remarkable example is the wellknown 1956 event $[1,2]$, when a 50-fold increase in the neutron background on the Earth's surface was detected. It has been shown that corresponds increase at aircraft altitudes was as high as 300 -fold. The areas near the polar regions are the least protected from the invasion of energetic charged particles. Events similar to one in 1956 possess the risk of significant radiation exposure of the crew and passengers the commercial flights, which pass near the northern polar region.

It should also be noted that variations of the charged particle fluxes in near-Earth space are usually accompanied by disturbances in the magnetospheric-ionospheric plasma and electromagnetic environment, which affect the propagation of radio waves and, accordingly, the quality of radio communications.

Another issue for the spacecraft is due to electromagnetic transients, i.e. intense shortterm increases in electromagnetic radiation fluxes. They can be observed in almost all spectral ranges - from radio to gamma. The nature of these phenomena is not fully understood yet. They can be generated in the upper atmosphere and are associated with high-altitude atmospheric discharges. These processes are accompanied by explosive energy release in about tens of microseconds. As a result, charged particles, in particular, electrons, can be accelerated to energies of tens of $\mathrm{MeV}$ and hard X-ray and gamma-ray bursts, high-energy electron beams, high-power electromagnetic pulses as well as possibly protons and neutrons can be generated [3]. Recent experiments on neutron detection during a thunderstorm have shown that during lightning discharges, electrons can be accelerated to energies, which are sufficient to generate bremsstrahlung gamma rays, which initiate photonuclear processes [4]. The possible dangerous effect on aircraft and human organisms of transient atmospheric phenomena and associated radiation remains completely unexplored. In principle, such events can harm the crew of the plane, which flies close (within tens of kilometers) to the source, and the electromagnetic pulse generated in the transient event, as well as energetic particles, can lead to malfunction of the onboard electronics with unpredictable consequences.

One of the ways to minimize the negative consequences of such events is the nowcasting of the radiation environment. The most common approaches to the nowcast are direct radiation measurements and model calculations based on ground and satellite measurements. Satellite measurements, which are available now, cover a limited number of orbits and a range of pitch angles (the angle between the particle velocity vector and the 
magnetic field line) and cannot provide a global picture of the space-time variations of radiation in near-Earth space. In the view of the problems described above, the real-time monitoring of radiation environment, forecasting dynamics of the radiation, and immediate risk assessment are necessary. These are the primary motivation for Universat-SOCRAT project [5]. With this project, we plan to create a constellation of small satellites and CubeSats. In addition to the monitoring of the radiation environment and electromagnetic transients (cosmic gamma-ray bursts, optical, ultraviolet and gamma-ray flashes from the Earth's atmosphere), dangerous objects of the natural (asteroids, meteors) and technogenic origin (space debris) would be tracked.

\section{Multi-satellite experiment for monitoring of hazard phenomena in the space and atmosphere}

Minimization the adverse effects of the described phenomena on the spacecraft and aircraft requires a real-time monitoring system of space radiation and atmospheric transients phenomenon. The system should provide up-to-date information on the dynamics of potentially unfavourable factors along the satellite orbits and aircraft flight paths. Basing on the measurements, it should issue recommendations on trajectory optimization or electronics functioning according to the risk level. The most effective way to create such a system is to use a multi-satellite constellation, including small and ultra-small satellites of the cubesat type.

\subsection{Concept of experiment}

In the Universat-SOCRAT project, effective monitoring of radiation in near-Earth space and on flight paths of aircraft should be implemented for the first time. It would use realtime data of charged particle and gamma-ray fluxes, as well as the results of observations of electromagnetic transients in the Earth's atmosphere. Group of spacecraft in different orbits would simultaneously cover both low-latitude and high-latitude regions of near-Earth space. An interactive model would use solar and geomagnetic activity parameters and measured data for calculating radiation environment in the near-Earth space and on aircraft flight paths and account.

Within the framework of the Universat-SOCRAT project, several small spacecraft should be launched on specially selected orbits. In the test version, the group of satellites should consist of three spacecraft. One spacecraft of medium mass (small satellite) should be launched on a low solar-synchronous orbit with an altitude of about 500-650 km and an inclination of $97-98^{\circ}$. The other two satellites are CubeSats. One of them would take almost circular orbit with an altitude of about $1500 \mathrm{~km}$ and an inclination of $\sim 80^{\circ}$. Another one would operate on an elliptical orbit with an apogee of about $8000 \mathrm{~km}$, a perigee of 600 $700 \mathrm{~km}, 63.4^{\circ}$ inclination, and $\sim 310^{\circ}$ argument of perigee.

The largest satellite payload should include instruments for monitoring of space radiation, a set of tools for visual tracking of hazardous objects, a set of devices for studying of atmospheric phenomena in the optical range, a group of instruments for observation in gamma-ray range, and a special unit for data collection. The payload should also include three-component magnetometer. The payload of CubeSats should include radiation particle spectrometer, a compact gamma spectrometer, an optical camera with a wide field of view, an ultraviolet detector and an electronics unit for data collection. The payload of all three satellites also should include the special electronic unit for data collection from detector units, its transmission to the board systems and feeding to the instruments power supply and commands. 
The test multi-satellite group can be extended with nano-satellites of the CubeSat type. The relative cheapness and simplicity of manufacture allow carry out more or less regular launches of satellites as a secondary payload. The constellation of nano-satellites will significantly increase the efficiency of space radiation measurements and monitoring of the transient electromagnetic events.

A couple of CubeSats with several multidirectional proton and electron spectrometers will provide data for elaboration of a three-dimensional dynamic model of radiation environment in the near-Earth space. For this, the satellites should be launched onto specially selected orbits and provide measurements that will allow us to calculate the current distribution of particle fluxes in a large volume of near-Earth space, i.e. from low orbits to geostationary, and, as a result, determine the current levels of radiation doses for a wide range spacecraft orbits, and also give a forecast of the radiation situation at low altitudes. Simultaneous measurements of particle fluxes on several spacecraft located at different points in near-Earth space will also allow sufficiently reliable separation of temporal and spatial effects in detected variations of the instrument output readings.

The CubeSat constellation also gives an advanced dataset to understand the physical nature of electromagnetic transients. The key question is confirming or refuting their direct connection with the thunderstorm or lightning. This problem requires the precise timing of the event and localization of the transient source. The angular resolution provided by a single instrument on a single satellite is not better than $\sim 5-10^{\circ}$ even for intensive events. It limits the spatial accuracy of transient source localization of $\sim 100 \mathrm{~km}$, if the observation is done at the altitude of $500 \mathrm{~km}$. It is enough to attribute the source to a thunderstorm area, but it is insufficient to attribute to a thunderstorm cloud. This latter task will demand an improvement of the angular resolution by at least a factor of 10. An alternative approach is the triangulation method, which combines observations of the selected area from different satellites. For this purpose, the number of CubeSats should be launched into similar orbit for the joint observations of a given area. The triangulation approach requires maintaining the given distance between satellites. This technical problem can be solved using thrusters.

For all spacecraft in the constellation, the regular operational mode of the instruments is the on-duty mode, when all devices are switched-on and operate continuously. Instrument switching between the operating modes is carried out by commands coming from the Earth or by the internal cyclograms. To optimize the payload energy consumption, the data exchange between instrument electronic and satellite board should be foreseen, including information on changing the parameters of the spacecraft power system and payload switching into energy-saving modes (changes in the instrument operating modes or their partial switching-off).

\subsection{Instruments for radiation monitoring}

Instruments for the radiation monitoring include proton spectrometer operating in the energies from 2 up to $>160 \mathrm{MeV}$ and electron spectrometer with energies of $0.15-10 \mathrm{MeV}$. The spectrometer is designed as a telescope with a few semiconductor (silicon) detectors with different thickness and a scintillator detector. All sensors are placed co-axially one after another. Measurements of the pitch-angle distribution and omnidirectional fluxes require several telescopes with differently directed axes. It is also discussed the instrument arrangement, in which axes of four telescopes are laying in the magnetic meridian plane and the axis of one other telescope is normal to this plane. In the case of polar orbit, it means that the axis of 4 telescopes should lie in the orbital plane.

The spacecraft orbits on which instruments for radiation monitoring will be installed should provide coverage of the entire spatial volume of the observed radiation captured in a 
magnetic field, i.e., radiation belts. The payload should also include three-component magnetometer to estimate pitch-angles of detected particles.

Measurements of omnidirectional fluxes of captured particles should be provided, followed by model interpolation and extrapolation of the measured fluxes to the entire area of radiation belts. Such measurements will allow us to calculate the current distribution of particle fluxes in a large volume of radiation belts and, as a result, the current levels of radiation doses for a wide range of operating orbits.

In the Universat-SOCRAT mission, several proton and electron spectrometers should measure fluxes along the special orbits. The data allows predicting the current radiation particle distribution in the large volume of radiation belts from the low-Earth orbit up to geostationary orbit. Radiation particle fluxes can be used for fast calculation of the radiation dose rates in a wide range of satellite orbits.

Collected data would be processed with a fully automated ground-based satellite data operational analysis system developed at Moscow State University, which is designed to evaluate and predict radiation particle fluxes in near-Earth space in real-time.

\subsection{Instruments for electromagnetic transient monitoring}

\subsubsection{Instruments for electromagnetic transient monitoring in ultraviolet and optics}

Instruments for study transient luminous events (TLEs) in ultra-violet (UV) and optical bands should include position-sensitive spectrometer. It can be made using a small lens telescope with high time resolution for spectral measurements of TLE and lightning and UV and infra-red (IR) detector-photometer. At the current stage of the project, we use a detector, which is similar to the one used in Tatiana-2 and Vernov missions [6, 7]. For the latter satellite, the instrument will be improved by adding signal channels for the far UV range. Similar instruments allow comparison new data with data of previous experiments on study UV flashes in the atmosphere. Spectral measurements are necessary to determine the type and altitude of TLE as well as to reveal lightning discharges by typical $777 \mathrm{~nm}$ line and by the absence of signal in the range of oxygen absorption lines about $762 \mathrm{~nm}$. Axes of both instruments should be directed toward nadir with open angles of about $90^{\circ}$.

The TLE observations in the UV range from CubeSats will be done either with a compact Atmospheric Ultraviolet Radiation detector AURA-2 with a wide field of view or with its improved version AURA-2T. The first version of the AURA detector already launched on-board the VDNH-80 satellite. This instrument uses SiPM MicroFC-60035SMT silicon photomultiplier tubes (PMTs)with a sensitive range from 300 to $800 \mathrm{~nm}$, with the maximum spectral sensitivity at $420 \mathrm{~nm}$. The sensitive area is $6 \times 6 \mathrm{~mm}$ and the quantum efficiency of about $41 \%$ at $420 \mathrm{~nm}$. Silicon PMTs differs from traditional PMTs, which are used for ultra-fast photometry. The main advantages of silicon PMTs, which make them preferable for application on CubeSats, are compact (thickness about $1 \mathrm{~mm}$ ), low voltage $(25-70 \mathrm{~V})$, and low weight. The main advantages of the new detector are the high temporal ( $1 \mathrm{mcs}-10 \mathrm{~ms}$ ) and spatial resolution, higher sensitivity due to the larger area of the optical system.

The simultaneous operation of the detectors on different spacecraft in the monitoring mode will make it possible to control transient activity in the Earth's atmosphere, both of thunderstorm and extra-thunderstorm nature with large exposure. Cartography and monitoring of UV transients is an important task for understanding the interconnections of various energetic processes in the atmosphere. The quasi-stationary UV glow of the atmosphere can be an additional indicator of the state of the geomagnetic situation and the effect of the solar wind on the atmosphere and magnetosphere of the Earth. 


\subsubsection{Instruments for gamma transient monitoring}

In the Universat-SOCRAT project, the Terrestrial Gamma Ray Flashes (TGFs) would be studied with two instruments: the gamma-ray flash monitor (GFM), and a tracking gammaray spectrometer (TGS) of the high resolution and sensitivity. In addition to gamma-rays, the spectrometer is sensitive to high-energy (from $1 \mathrm{MeV}$ up to dozens of $\mathrm{MeV}$ ) neutrons [8]. The separate unit for data analysis and control of GFM and TGS should also be foreseen in the payload. This unit should contain digital electronics unit that will provide the record of the data stream with a time resolution of $\sim 10$ us, and generate the trigger signals for the detection of different type events, including TGFs and neutron flux increases. The data record must be referenced to the UTC time with an accuracy of $\sim 10$ us to allow the positioning of the TGFs, if observed simultaneously by several space missions, using triangulation, and for the comparison with the data of ground-based lightning nets.

We use X-ray and gamma-ray detectors for TGF triangulation. The detector do not have spectrometer capabilities to satisfy mass requirements for CubeSats. Each of CubeSats carry at least 1 set of gamma-ray detectors; the $6 \mathrm{U}$ CubeSat has at least three of them.

If the gamma-ray detector is the only one on the CubeSat, its axis should be directed along the nadir - zenith axis to nadir and the field of view should not be obscured within \pm $60^{\circ}$ from the observation direction. In the case of two instruments installed on the CubeSat, their axes should be perpendicular to each other, and the axis of one detector should be directed to nadir and the axis of the other detector should be directed along the spacecraft velocity vector in the case of the orbital orientation of the spacecraft (along the line "zenith - nadir" and the velocity vector), while the field of view should not be obscured within \pm 60 from the axis of the detector. If three instruments are installed on the spacecraft, their detector axes should be perpendicular to each other, directed along mutually perpendicular edges of the cube, as if forming a Cartesian coordinate system, while the axis of one detector should be directed to the nadir, the other along the velocity vector, the axis of the third should complement the three-axial coordinate system to complete.

Gamma-ray detector should be installed in a CubeSats in such a way that the surface of its input window coincides with the surface of one of the face-side of the satellite. The instrument should consist of detector assemblies and electronics boards. We consider different options of the detector units. In the basic version, we consider four detector units, which include scintillatior crystals with a size of $45 \times 45 \times 5 \mathrm{~mm}$ and a photo-detector. The possible scintillators candidates are CsI(Tl) crystals (a low-cost option) or Ce: GAGG (an optimal option). For the signal pickup, photodetectors such as PMTs or silicon photomultipliers (Si-PM) can be used.

To distinguish different types of space radiation (electrons, gamma-rays), a fosvich detector setup can be used. In a fosvich detector a plastic or inorganic scintillator (CsI (Tl) or Ce: GAGG), is viewed by a single photodetector. To identify the different particle types the separation of signals from different scintillators is carried out by analyzing the time profiles of the PMT output signals.

Alternatively, a multi-pixel version of the detector can be used. It is build of individual crystals (pixels) of relatively small size each with its own photodetector: an array of standard silicon PMTs, or self-designed photodetectors and electronics.

\section{First results of Moscow university CubeSat missions}

The first stage of the Universat-SOCRAT program started on July 5, 2019 with a successful launch of three CubeSats (SOCRAT, AmurSat, VDNH-80) from the Vostochny cosmodrome. These satellites are equipped with Detector of Cosmic Radiation (DéCoR) instruments for monitoring space radiation and prototypes of instruments for observing 
transient phenomena in the Earth's atmosphere. The prototypes are scintillation fosvich detectors, which are designed for gamma transient observation and installed on AmurSat and VDNH-80. These instruments detect charged particles and gamma-rays in the energy release range of $0.1-2.0 \mathrm{MeV}$. The geometric factor of these instruments is $\sim 50 \mathrm{~cm} 2 \mathrm{sr}$. VDNH-80 CubeSat also contains an optical photometer AURA, which uses four silicon photomultipliers covered by different light filters. Thus, the instrument observes the Earth's atmosphere in four spectral bands from ultraviolet to red. The satellites operate at a sunsynchronous orbit with an altitude of $\sim 800 \mathrm{~km}$. These orbits provide good conditions for monitoring space radiation in various areas of near-Earth space, including zones of trapped radiation, areas of precipitation, and etc. Such an orbit also allows observations of flash phenomena both in the near-equatorial and high-latitude regions.

\subsection{Parameters, structure and functioning principles of DeCoR payload}

The DéCoR instrument is designed for measuring the fluxes and spectra of charged particles and gamma-rays in the energy release range of $0.1-2 \mathrm{MeV}$. The main scientific objectives are the study of fast variations of electron fluxes in the areas of precipitation and the slot region between radiation belts, and the study of the dynamics of particle fluxes and gamma-rays at low-Earth orbits under different geomagnetic conditions. An important factor providing an advanced scientific research is the installation of the similar instruments on two satellites, sequentially flying through the same region of near-Earth space. Figure 1 shows the DeCoR instrument. Technical parameters of the DeCoR instrument are listed in Table 1.

The DeCoR instrument is designed in order to be used in the CubeSats missions. The detector is a two-layer assembly of a plastic scintillator and a CsI (Tl) crystal (see Figure 2). The detector has a sensitive area of $\sim 18 \mathrm{~cm} 2$. The assembly is viewed by two PMTs. Gamma-rays and electrons can be identified by analyzing the time profile of the signal pulse. In order to do the analyze, separate digitization of the initial and final parts of the PMT output pulse ("fast component" and "slow component") is done for each of PMTs. Also, the scintillator in which the interaction took place can be determined.

Instrument's microcontroller is responsible for data and commands exchange with the satellite's onboard systems (the UART protocol). Non-volatile memory with a capacity of $16 \mathrm{MB}$ keeps the scientific data. For transmission to the Earth, the DeCoR instrument generates both monitoring data (particle counting rates 1 time per second) and detailed data about all interactions in the detector with a time resolution of $20 \mathrm{mcs}$ (so-called event-byevent mode), which are accumulated in the instrument's memory by a command that determines the moment of interesting time from the view of the researcher. The main way to conduct a scientific experiment is regular collecting and transmitting monitoring data to the Earth, as well as activation from time to time the detailed recording mode on specialy selected parts of orbit, where variations of the trapped and quasi-trapped particle fluxes are possible. 


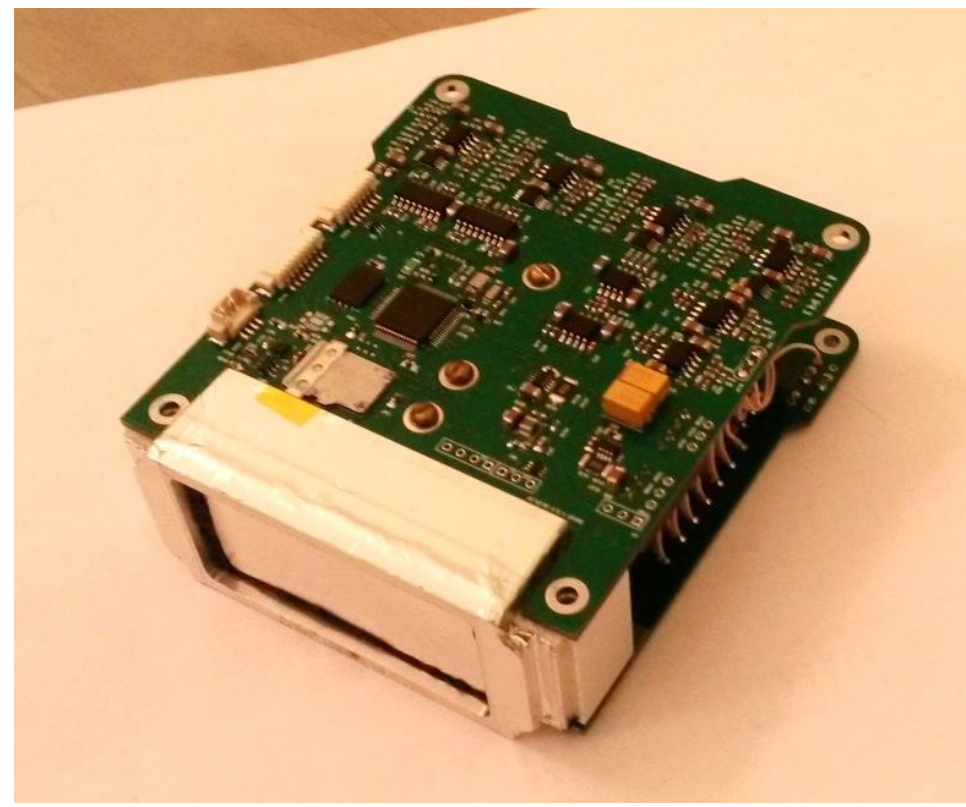

Fig. 1 The general view of DeCoR instrument.

After analyze of data of the "monitoring" type, the researcher identifies the time points at which the flux variations have occurred. For these periods, detailed data is requested from the CubeSat. The data transfer rate allows one to transmit detailed data about an interesting phenomenon, for example, about the precipitation of magnetospheric electrons lasting several minutes, for 5 - 10 days (when using one receiving station).

Table1. Parameters of DeCoR instrument

\begin{tabular}{|l|c|}
\hline Types of detected particles and quanta & Gamma quanta, electrons \\
\hline Range of energy release, $\mathrm{MeV}$ & $0.1-2.0$ \\
\hline Effective area, $\mathrm{cm}^{2}$ & 18 \\
\hline & \\
Dynamical range: & $0-1000$ \\
Monitoring of fluxes, $\mathrm{cm}^{-2} \mathrm{~s}^{-1}$ & $0-25$ \\
Measurements in event by event mode, $\mathrm{cm}^{-2} \mathrm{~s}^{-1}$ & \\
\hline Time resolution, mcs & 20 \\
\hline Size, mm & $102 \times 90 \times 36$ \\
\hline Mass, $\mathrm{g}$ & 400 \\
\hline Voltage, $\mathrm{V}$ & 7.5 \\
\hline Power consumption, $\mathrm{W}$ & 0.7 \\
\hline
\end{tabular}




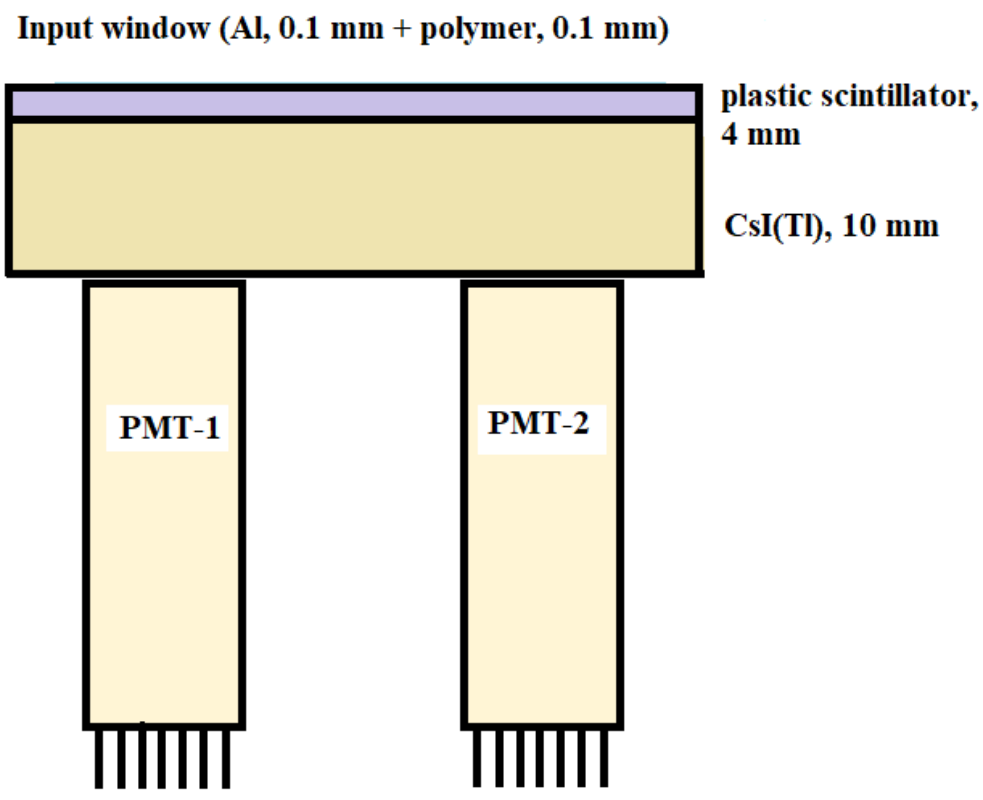

Fig. 2. The DeCoR detector unit.

The DeCoR instrument includes a magnetometer, which makes it possible to measure the magnitude of the magnetic field along three mutually perpendicular axes. Data on the magnetic field allows account the spacecraft spinning in the study of trapped and precipitating electrons and even use the data to reconstruct the pitch-angle distribution of particles. The magnetometer data is included directly in the monitoring information frames, which contain the parameters of the measured radiation.

\subsection{First results of observations with DeCoR instrument on-board AmurSat}

Figure 3 shows an example of the DeCoR data obtained from AmurSat CubeSat on August 14, 2019. It includes the satellite geographical position and evolution (1 s time resolution) of counting rate in the plastic detector (mainly electrons), $\mathrm{CsI}(\mathrm{Tl})$ (mainly gamma quanta) and total count rates.

The maxima in time dependencies on the figure 3 correspond to the moments when the satellite was passing the outer radiation belt of the Earth. During the flight near the equator $(12: 00-12: 18)$, there is a smooth increase in electron fluxes, while the counting rate of background gamma-rays is constant. 

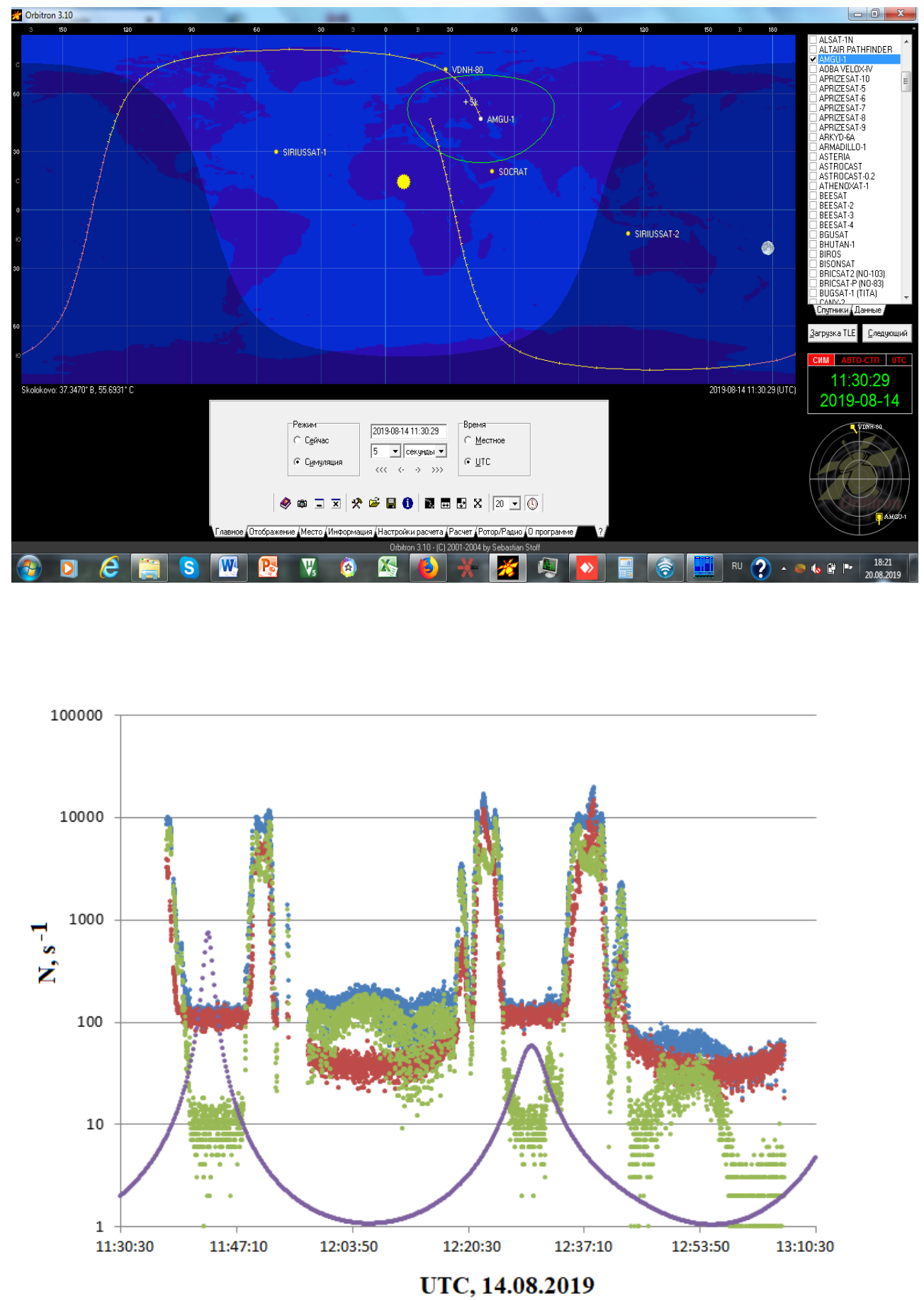

Fig. 3. Top panel: AmurSat orbit projection on the map. Bottom panel: time dependences of counting rate during one orbit in plastic scintillator (green points), $\mathrm{CsI}(\mathrm{Tl})$ (red points) and total (blue points). The dark blue line represents the corresponding McIlwine parameter (L). 


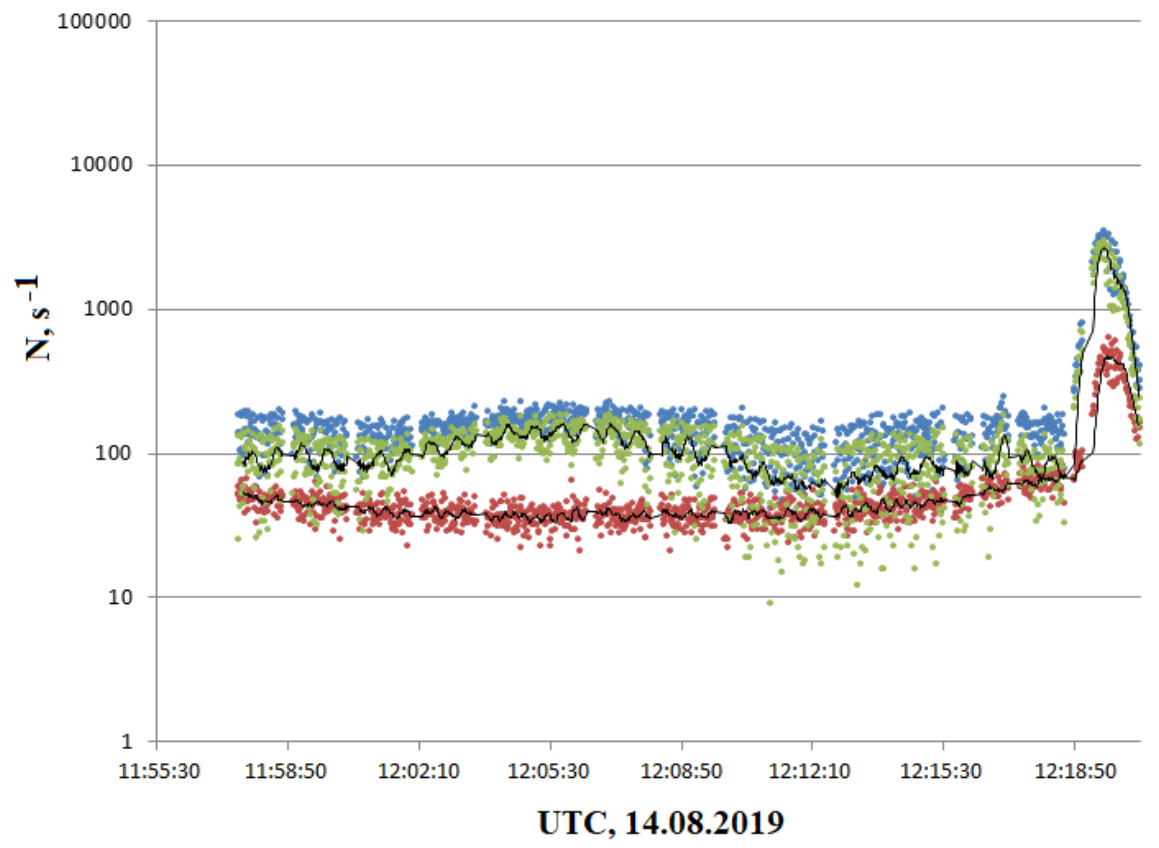

Fig. 4. Monitoring counting measured near Equator 14.08.2019 in plastic scintillator (green points), $\mathrm{CsI}(\mathrm{Tl})$ (red points) and total (blue points). The dark lines represent the moving averages of corresponding counting rates.

The data of the DeCoR instrument near the equator are shown in Figure 4 with a higher time resolution. The average values of the counting rates in $\mathrm{CsI}(\mathrm{Tl})$ and in plastic scintillator also presented in this figure as solid lines. The line approximated the averaged counting rate of events in plastic (electrons with energies less than $700 \mathrm{keV}$ ) shows the modulation with a period of $\sim 40 \mathrm{~s}$, which caused by the rotation of the satellite. For events in $\mathrm{CsI}(\mathrm{Tl})$ (mainly gammas), the modulation is practically not observed. Thus, we can conclude that near the equator we have observed electrons with non-isotropic flux distribution.

Figure 5 shows electron and gamma-ray fluxes, which have been measured during one orbit turn on August 30, 2019. The satellite was in the same orbit as on August 14 (see Figure 5). Comparison of the counting rates shown in Figure 3 and Figure 5 demonstrates the similarity of the main typical features, i.e. maxima corresponding to the crossing of the outer radiation belt, narrow maxima observed symmetrically near the inner boundary of the belt. The broad maximum near the equator in Figure 5 is also observed, while its shape does not completely coincide with Figure 3, which may be the subject of further research. 


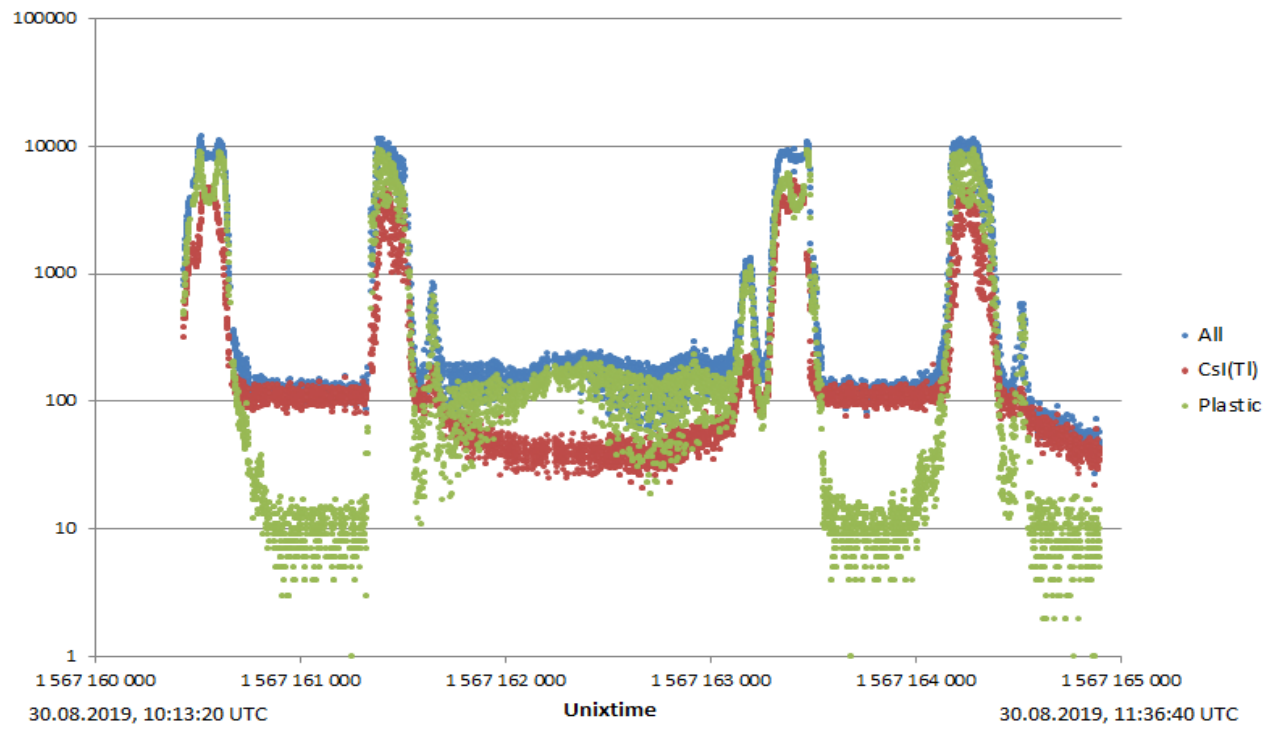

Fig. 5. Monitoring counting measured 30.08.2019 during one orbit in plastic scintillator (green points), $\mathrm{CsI}(\mathrm{Tl})$ (red points) and total (blue points).

\subsection{First results of observations with DeCoR instrument on-board VDNH-80 cubesat}

Figure 6 shows the evolution of counting rates in two channels of the DeCoR instrument onboard the VDNH-80 CubeSat. The measurements took place during November 2, 2019. For this session the rate of output parameter records was pre-set using the control command as one time per $0.3 \mathrm{~s}$ (the default value is $1 \mathrm{~s}$ ), i.e. these dependencies were recorded with $0.3 \mathrm{~s}$ time resolution. Figure 6 also shows the satellite orbit projection during this period on the world map. The satellite moved from an area close to the North Pole to the south toward North America. At the beginning of the considered orbit, it was in the northern polar cap, after which it crossed the region of the outer radiation belt. This moment corresponds to a maximum with a high value of $\sim 12000 \mathrm{~s}^{-1}$ of counting rates.

The modulation of the counting rates observed during the satellite flight through the region of increased electron fluxes is due to the rotation of the satellite. At moments when the angle between the axis of the instrument and the direction of the magnetic field is small, minimums of the counting rate should be observed, since there are fewer particles with similar pitch angles, since they drop out, reaching the Earth's atmosphere. 

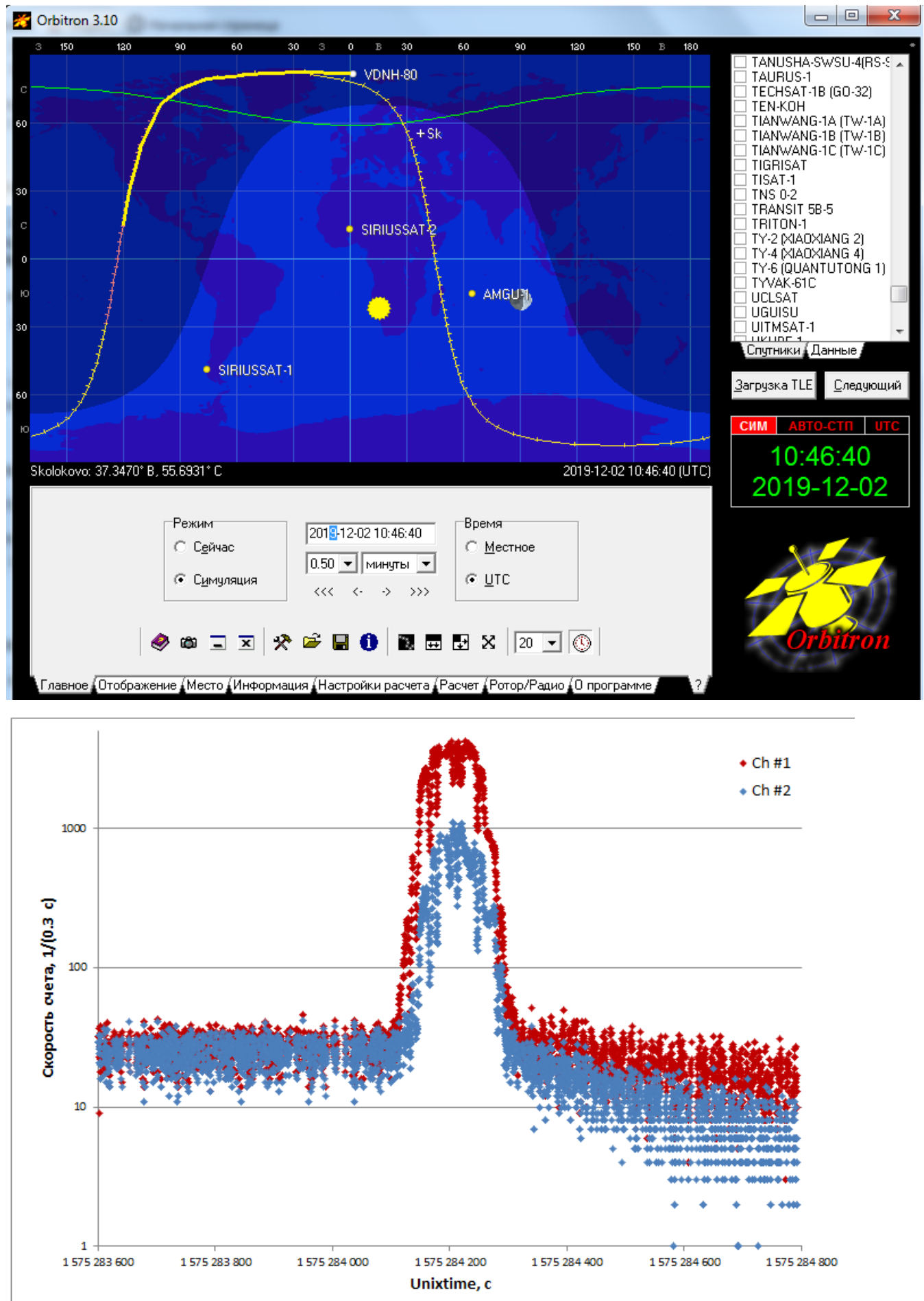

Fig. 6. Top panel: VDNH- 80 orbit projection on the map. Bottom panel: time dependences of counting rate during one orbit in $\mathrm{CsI}(\mathrm{Tl})$ (blue points) and sum in plastic and $\mathrm{CsI}(\mathrm{Tl})$ (red points). 


\section{Plans of Moscow university CubSat launching in 2020}

We plan to launch three more CubSat spacecraft during 2020. One of them, the $6 \mathrm{U}$ DECART satellite, should contain three DeCoR instruments, as well as an improved AURA-2 photometer. The DeCoR instrument should also be installed on a $6 \mathrm{U}$ satellite, being developed jointly with the Novosibirsk State University, as well as on a $1.5 \mathrm{U}$ spacecraft, being developed jointly with the N.E. Bauman Moscow State Technical University.

The DeCoR instrument is the same as ones, which were installed on-board AmurSat and VDNH-80 CubeSat satellites. It is a CsI(Tl) / plastic scintillation phosphate detector that registers charged particles and gamma quanta in the energy release range $0.1-2.0 \mathrm{MeV}$. The geometric factor of the device is $\sim 50 \mathrm{~cm}^{2} \mathrm{sr}$. The optical photometer AURA-2 is made on the basis of silicon photomultiplier arrays, whose entrance windows are covered with different light filters.

The axes of the three DeCoR instruments installed on the DECART satellite should be normal to each other and directed in such a way, that one to the nadir, the second to the spacecraft velocity vector, the third to the axis complementing the zenith-nadir line and the velocity vector to the right coordinate system. This configuration will make it possible to estimate the anisotropy of the detected electron fluxes and identify precipitated particles. The AURA-2 instrument should be directed to the nadir and perform monitoring observations of the UV emission of the Earth's atmosphere, including detecting electromagnetic transients.

In the case of a successful launch of these spacecraft, a unique multi-satellite constellation will be implemented for the first time, allowing simultaneous measurements of particle and quantum fluxes using the same type of instruments at different points in near-Earth space. Such measurements will provide unique information on the nature of variations, including short-term (with characteristic times less than $1 \mathrm{~ms}$ ), fluxes of electrons of sub-relativistic energies in near-Earth space. In addition to the applied aspects related to monitoring space weather, such measurements will provide important data necessary for understanding the mechanisms of acceleration and loss of trapped and quasitrapped electrons.

\section{Conclusion}

The first stage of this project began with the launching of three 3U CubeSats in July 2019. The satellites carry the prototypes of the instruments, which would be used in the following "Universat-SOCRAT" missions. Collected data confirmed that the instruments operate properly, and detector parameters have been selected correctly. The particle flux measurements at low polar orbit, which have been carried out during flight tests, demonstrate the expected behaviour. The DeCoR instruments on-board AmurSat and VDNH-80 CubeSats have successfully measured particle flux in continuous (1 s resolution) and event-by-event (20 mcs time frame) modes. We have used these data to analyse both slow and fast particle flux variations. Finally, flight tests have confirmed the suitability of the instruments for the scientific research for which they were intended.

The next three CubeSats for the Universat-SOCRAT programme are equipped with the same instruments as AmurSat and VDNH-80. Their launch is scheduled for 2020.

The Universat-SOCRAT project could become the first space-deployed system for monitoring various space hazards for aviation, sub-orbital, and orbital flights. The next steps in the project would address the following problems:

- the radiation environment now-casting for near-Earth space and radiation risks assessment for space missions and the producing alert signals for mission control centres; 
- the radiation environment nowcasting at the aviation altitudes and radiation risks assessment for crew members;

- verification of models of the radiation environment in the near-Earth space;

- real-time tracking of potentially dangerous objects of natural and technogenic origin near the Earth;

- detection of electromagnetic transients in the upper atmosphere and space.

\section{Acknowledgments}

Financial support for this work was provided by the Ministry of Education and Science of Russian Federation, Project № RFMEFI60419X0237.

\section{References}

1 T. Gold and D. R. Palmer, J. Atmosph. Terr. Phys., 8, 287-290 (1956)

2 P. L. Marsden, J. W. Berry, P. Fieldhouse and J. G. Wilson, J. Atmosph. Terr. Phys., 8, 278-281 (1956)

3 M. Casolino, P. Klimov and L. Piotrowski, Progress of Theoretical and Experimental Physics. 2017, 12, p. 12A107 (2017)

4 T. Enoto, W. Yuuki, F. Yoshihiro et al., Nature. 551, 481 - 485, (2017)

5 V. I. Osedlo, V. A. Sadovnichii, M. I. Panasyuk et al., Advances in the Astronautical sciences. 163, 45-52 (2018)

6 G. K. Garipov, B. A. Khrenov, P. A. Klimov et al., J. Geophys. Res. 118(2), 370-379 (2013)

7 M. I. Panasyuk, S. I. Svertilov, V. V. Bogomolov et al., Adv. Space Res. 57, 835-849, (2016)

8 M. Panasyuk, P. Klimov, S. Svertilov et al., Progress in Earth and Planetary Science. 6, 35, (2019) 